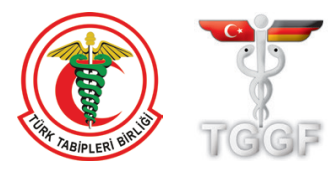

\title{
A short review of current implementations of sentinel lymph node mapping in gynecologic cancers
}

\author{
(D) Duygu Altın 1 , (D) Salih Taşkın², (D) Fırat Ortaç² \\ ${ }^{1}$ Clinic of Gynecology and Obstetrics, Ordu University Training and Research Hospital, Ordu, Turkey \\ 2Department of Gynecology and Obstetrics, Ankara University Faculty of Medicine, Ankara, Turkey
}

\section{Abstract}

Lymph node metastasis both increases disease stage and alters adjuvant treatment plans in gynecologic cancers. Since a minority of the patients have nodal metastasis, many patients unnecessarily undergo complete lymphadenectomy and are exposed to the subsequent morbidities. Sentinel lymph node (SLN) mapping is an alternative for evaluation of lymph nodes with lesser side effects. Although it is yet an experimental approach in ovarian cancer, it has been incorporated into guidelines for endometrial, cervical and vulvar cancers. We aimed to summarize the current situation of SLN mapping in gynecologic cancers. (J Turk Ger Gynecol Assoc 2021; 22: 242-8)

Keywords: Sentinel lymph node, endometrial cancer, cervical cancer, vulvar cancer, ovarian cancer

Received: 1 February, 2021 Accepted: 17 May, 2021

\section{Introduction}

Lymphadenectomy is a part of surgical staging in gynecologic cancers since it is important to determine the nodal status for guiding adjuvant treatment. However, only a minority of the cases have nodal metastasis and many patients face morbidities associated with complete lymphadenectomy unnecessarily. Sentinel lymph node (SLN) mapping has been proposed as a less invasive technique used for assessment of lymph nodes in gynecologic cancers. The lymph node that has direct connection to the tumor site, and most likely to receive any metastasis first is called the SLN. Therefore, with SLN mapping while nodal metastasis is identified, morbidities of complete lymphadenectomy can be avoided in node negative patients.

SLN mapping has gained importance in staging of gynecologic cancers in the last decade and it has been incorporated into National Comprehensive Cancer Network (NCCN) Guidelines for endometrial, cervical and vulvar carcinomas (1-3). Currently, in ovarian carcinoma SLN mapping remains an experimental approach.

Technetium-99m (99mTc), indocyanine green (ICG) and blue dyes can be used alone or combined for identifying SLNs. All suspicious lymph nodes must be removed besides SLNs and side-specific lymphadenectomy should be performed in case of mapping failure.

SLN mapping allows detection of uncommon drainage sites, such as internal iliac lymph nodes, that may not otherwise have been resected. Another advantage of SLN mapping is detection of more nodal metastasis by pathologic ultrastaging which cannot be identified by routine hematoxylin and eosin (H\&E) staining. To do so, SLNs are cut at 50-200 $\mu \mathrm{m}$ intervals and two paraffin embedded slides are prepared from each section. One slide is stained with $\mathrm{H} \& \mathrm{E}$ and the other with immunohistochemistry stains (AE1 and AE3 anticytokeratin antibodies) if no metastasis is identified by H\&E examination. Tumor deposits $>2 \mathrm{~mm}$ are defined as macrometastasis. Micrometastasis is defined as metastatic deposits ranging from $0.2 \mathrm{~mm}$ to no more than $2 \mathrm{~mm}$ and isolated tumor cells (ITCs) are defined as single tumor cells or clusters $<0.2 \mathrm{~mm}$.

Although it has been shown that the SLN algorithm is a highly sensitive method and has high detection rate and negative predictive value (NPV), survival data comparing only SLN removal and complete lymphadenectomy is insufficient (4-6). 
This review aims to summarize current SLN mapping implementations in gynecologic cancers.

\section{Endometrial cancer}

Endometrial cancer is surgically staged, which includes hysterectomy with or without oophorectomy and lymphadenectomy. Nodal status not only changes the stage of the disease but also guides the adjuvant treatment plans. However, most endometrial cancer patients are diagnosed in the early-stage when the disease is limited to the uterus and many patients undergoing complete lymphadenectomy face intraoperative and postoperative complications (eg. neurovascular injuries, lymphedema and lymphocele formation) unnecessarily $(7,8)$. Moreover, two randomized studies have shown that lymphadenectomy does not provide survival advantage in early-stage patients $(9,10)$. Therefore, the approach to lymph node evaluation is an issue of debate.

Many centers perform selective lymphadenectomy as the uterus is sent for intraoperative frozen section evaluation and no further lymphadenectomy is performed when the analysis reveals grade 1 or 2 tumors invading less than $50 \%$ of the myometrium and smaller than $2 \mathrm{~cm}$ (11). These criteria are also known as the Mayo criteria and the risk of nodal metastasis in this low-risk population is less than 5\% $(11,12)$. However, only $20 \%$ of high-risk patients have nodal metastasis and many patients still undergo unnecessary lymphadenectomies (13). Moreover, accuracy of frozen section is higher in more experienced centers and not all centers may have a frozen section unit to guide the surgery $(14,15)$.

The SLN mapping algorithm is a less invasive technique for evaluation of nodal status. Both in prospective and retrospective studies, sensitivity and NPV of the SLN mapping algorithm were reported between $84-97 \%$ and $97-99 \%$, respectively (4,16-19). It has been shown that the SLN algorithm does not compromise overall detection of stage IIIC disease, both in low-risk and high-risk endometrial cancer patients (20,21). In a meta-analysis including 55 studies, bilateral and overall detection rate of SLN mapping were $50 \%$ and $81 \%$, respectively (4). With these promising data, the SLN mapping algorithm is also incorporated into the NCCN guidelines, despite a lack of randomized studies comparing survival outcomes of SLN mapping and complete lymphadenectomy (1).

$99 \mathrm{mTc}$, blue dyes (1\% methylene blue, $1 \%$ isosulfan blue or $2.5 \%$ patent blue sodium) or ICG can be used for lymphatic mapping. ICG requires a near-infrared camera for visualization, but it is shown to be superior to blue dye alone and equal to a combination of $99 \mathrm{mTc}$ and blue dye in terms of SLN detection (22). Whichever dye is used, $1 \mathrm{~mL}$ deep $(1 \mathrm{~cm})$ and $1 \mathrm{~mL}$ superficial (3-4 mm) cervical injections are made at 3 o'clock and 9 o'clock positions before hysterectomy. Although fundal or hysteroscopic injections lead to higher mapping rates in the para-aortic area, they do not provide higher detection rates in the pelvic area compared to cervical injection (23). Optimal detection of SLNs occurs 15-60 minutes after the injection. Besides all identified SLNs and any enlarged or suspicious lymph nodes should be removed. Side-specific lymphadenectomy is required if a hemipelvis does not map. Para-aortic lymphadenectomy is not an essential step in the SLN algorithm and can be performed at the surgeon's discretion (Figure 1).

Compared to complete lymphadenectomy, although fewer nodes are removed, 4.5-8\% additional lymphatic metastasis is detected with the SLN algorithm as a result of ultrastaging $(18,24)$. One step nucleic acid amplification assay (OSNA) is a new method for intraoperative SLN assessment which detects cytokeratin 19 messenger RNA in metastatic lymph nodes. Compared to classic ultrastaging, more nodal metastasis is detected by OSNA, but no nodal tissue is left for postoperative assessment. Despite this, the prognostic significance of these metastases detected by OSNA is not known $(25,26)$.

Currently, micrometastasis is regarded as node positive and managed accordingly, but prognostic significance of ITCs is unknown and adjuvant treatment of patients with ITCs is given according to primary tumor characteristics (27). Yet, more studies are needed to clarify the clinical relevance and treatment of patients with ITCs.

In some retrospective series, it has been shown that removal of SLNs alone does not have a negative effect on

Peritoneal and serosal evaluation
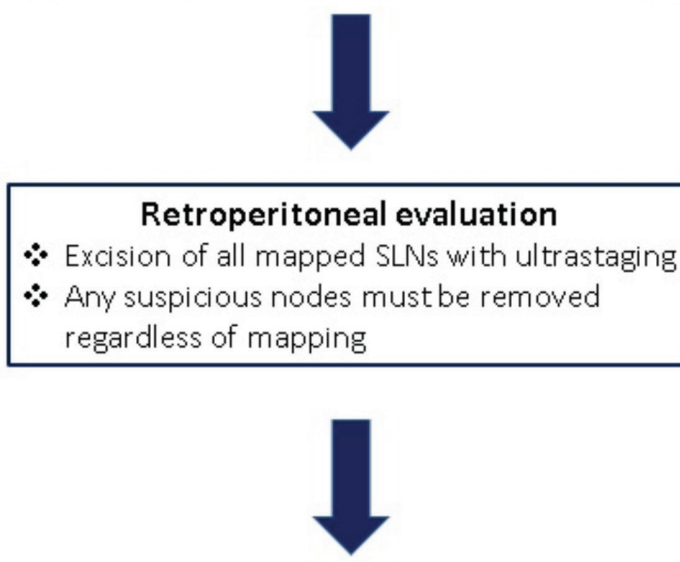

$\because$ If there is no mapping on a hemipelvis a
side-specific LND is performed
$\because$ Paraaortic LND is performed at the
attending's discretion

Figure 1. SLN mapping algorithm in endometrial cancer SLN: Sentinel lymph node, LND: Lymph node dissection 
oncological outcomes, both in low- and high-risk pathologies as 3-year overall survival and disease-free survival (DFS) were comparable between the SLN algorithm group and lymphadenectomy groups $(21,28-31)$.

\section{Cervical cancer}

Lymphatic metastasis has a negative effect on prognosis and alters treatment plans in cervical cancer. Sensitivity of preoperative imaging studies for nodal metastasis is reported below $75 \%$ and surgical evaluation is generally needed (32). The risk of nodal involvement is $<1 \%$ in stage IA1 cervical cancer and there is no need for lymphadenectomy in this stage when there is no lymphovascular space invasion. However, when the disease has been staged as IA2 and beyond, at least SLN removal is needed.

SLN biopsy in cervical cancer appears promising. Cervical injections are done with ICG, blue dye or 99mTC at two or four points (2). All enlarged and suspicious nodes must be removed in addition to the SLNs. Side-specific lymphadenectomy is mandatory if SLN is not detected. One hundred and thirty-nine women with stage IA1 to IB2 cervical cancer were recruited to the SENTICOL study, at least one SLN was detected in $98 \%$ of the patients, and sensitivity and NPV were $92 \%$ and $98.2 \%$, respectively (33). In another study, SLN detection rate, sensitivity and NPV were $88.6 \%, 77.4 \%$ and $94.3 \%$, respectively (34). Although the SLN technique can be used in tumors up to $4 \mathrm{~cm}$, the best detection rates, sensitivity and NPV are achieved in tumors smaller than $2 \mathrm{~cm}(5,34)$. Moreover, studies have demonstrated that SLN biopsy is completely reliable if bilateral SLNs are detected $(33,35)$.

Similar to endometrial cancer, ultrastaging is performed if no metastasis is identified in SLN with routine H\&E staining. A recent study showed that patients with micrometastasis and ITCs had similar DFS compared to node negative patients (92.7\% vs 93.6\%) and DFS did not improve with adjuvant treatment in these patients (36). Yet more studies are needed to clarify clinical significance and treatment of low-volume metastasis. The ongoing prospective randomized SENTICOL III and SENTIX studies aim to find out whether DFS, recurrence rate and quality of life differs between patients with cervical cancer undergoing only SLN biopsy and complete bilateral pelvic lymphadenectomy after SLN biopsy.

\section{Vulvar cancer}

Lymphatic metastasis is the most important prognostic feature in vulvar cancer and the 5-year disease specific survival for node positive and negative patients were reported between $70-93 \%$ and $25-41 \%$, respectively (37). The primary tumor should be resected with at least $1 \mathrm{~cm}$ clear margins and either unilateral or bilateral inguinofemoral lymph nodes or in selected patients SLNs should be removed. There is no need for inguinofemoral lymphadenectomy for stage IA patients since risk for nodal involvement is $<1 \%$ (38). But patients with stage IB or higher stages have $\geq 8 \%$ risk of nodal metastasis and lymphadenectomy is mandatory (39). Unilateral lymphadenectomy can be performed in case of clinically negative lymph nodes when the tumor is $<4 \mathrm{~cm}$ and $\geq 2 \mathrm{~cm}$ lateral to vulvar midline (3).

$20-70 \%$ of the patients experience surgical morbidities, such as wound breakdown and lymphedema, due to inguinofemoral lymphadenectomy (40). SLN biopsy is an option for inguinofemoral lymphadenectomy in selected early-stage patients whose tumors are unifocal and smaller than $4 \mathrm{~cm}$ and in whom imaging and/or clinical examination reveals negative lymph nodes $(41,42)$. For tumors $>4 \mathrm{~cm}$, the SLN technique is both associated with reduced sensitivity and higher groin recurrences (43). It is also recommended to perform complete inguinofemoral lymphadenectomy after SLN biopsy in at least 10 cases before performing SLN biopsy alone. The best SLN detection rates are observed when $99 \mathrm{mTC}$ and blue dye are used together $(42,44)$.

$99 \mathrm{mTC}$ is injected 2-4 hours prior to the operation and a gamma probe is used to detect the SLN. The most commonly used blue dye is isosulfan blue $1 \% .4 \mathrm{cc}$ blue dye is injected intradermally into the normal tissue around the tumor at 2, 5, 7 and 10 o'clock positions. It is recommended to perform SLN biopsy prior to excision of vulvar tumor so the lymphatic network will not be disturbed. Side-specific inguinofemoral lymphadenectomy is recommended when SLN is not detected.

In the GOG-173 study, SLN biopsy was prospectively compared to inguinofemoral lymphadenectomy in 452 patients with squamous cell carcinoma with tumors ranging between 2-6 cm in diameter and at least $1 \mathrm{~mm}$ depth of stromal invasion (42). At least one SLN was identified in 92\% of the cases, and sensitivity and NPV were $91.7 \%$ and 96\%, respectively. In another prospective study, the GROINSS-V I study, investigating clinical safety and utility of SLN biopsy in early-stage vulvar cancer, the false negative rate of SLN biopsy was found to be $3 \%$ (44). Four hundred and three women with stage I-II squamous cell tumors $<4 \mathrm{~cm}$ were enrolled in this study, and no further lymphadenectomy was performed if SLNs were negative. In a study evaluating oncologic outcomes of the GROINSS-V I study participants, groin recurrence rates for SLN negative and positive patients at 5 years were $2.5 \%$ and $8 \%$, respectively (45).

The ongoing GROINSS-V II study is a prospective study which aims to find out whether complete inguinofemoral lymphadenectomy can be replaced by adjuvant radiotherapy (RT) in patients with metastatic SLNs undergoing only 
SLN biopsy. Preliminary results of this study showed that inguinofemoral lymphadenectomy can be safely replaced by RT in patients with low-volume metastasis, but not for patients with macrometastasis (46). Therefore, when SLN metastases are $>2 \mathrm{~mm}$, it is recommended to perform complete ipsilateral lymphadenectomy. In such cases, contralateral lymph nodes should also be resected or treated with external beam radiation therapy. Frozen section of SLNs may be used to for deciding to perform complete lymphadenectomy.

The aim of GROINSS-V III will be to examine the effectiveness and safety of chemoradiation in patients with macrometastatic SLNs.

\section{Ovarian cancer}

In apparently early stage ovarian cancer, bilateral pelvic and para-aortic lymphadenectomy is indicated to detect occult lymphatic metastasis, since $1 / 3$ of these patients have nodal metastasis and these metastases can be seen in bilateral pelvic or para-aortic nodes $(47,48)$. It has been shown that earlystage ovarian cancer patients who had undergone systematic lymphadenectomy had better survival outcomes compared to no lymphadenectomy (49).

SLN biopsy is investigational in ovarian cancer. 99mTC, blue dye or ICG can be used as tracers alone or in combination (50). Whichever dye is used, the best detection rates were reported with injections at the utero-ovarian and infundibulopelvic (IP) ligaments, or only at the IP ligament if hysterectomy had been performed previously, just underneath the peritoneum. Mesovarium, ovarian hilum and ovarian cortex are alternative injection sites, but lower detection rates were reported, and the latter technique may result in tumor rupture.
In a systematic review including 145 patients, overall SLN detection rate was found to be $90.3 \%$ (range; $40-100 \%$ ) (50). While ICG alone resulted in $93.3 \%$ of detection rate, with ICG + 99mTC combination, it was $100 \%$. Mean detection rate in the pelvic and para-aortic region were $44 \%$ (range; $25-87.5 \%$ ) and $82 \%$ (range; $70-91 \%$ ), respectively. In a recent prospective cohort study including 20 patients, first unilateral salpingo-oophorectomy was performed and SLN mapping was performed with $99 \mathrm{mTC}$ plus $0.5 \mathrm{~mL}$ of ICG afterwards, if the frozen section revealed malignancy (51). Hence, injections were done into both utero-ovarian and IP ligament stumps or only into the IP ligament stump according to previous hysterectomy status. SLNs were identified in all patients in the para-aortic region and in $93 \%$ of the patients in the pelvic regions. The ongoing prospective SELLY trial is a phase II trial, which aims to evaluate accuracy of SLN biopsy in diagnosing nodal metastasis in early-stage ovarian cancer patients using ICG. The preliminary results of this study that included 31 patients revealed overall detection rate of $67.7 \%$ and detection rate was significantly higher in patients undergoing immediate staging surgery compared to delayed surgery. All four patients with lymphatic metastasis had metastatic SLNs, yielding $100 \%$ sensitivity and $100 \%$ NPV (52).

\section{Conclusion}

Table 1 summarizes the NCCN and European Society of Gynaecological Oncology recommendations for SLN mapping in gynecologic cancers.

Table 1. NCCN and ESGO recommendations for SLN mapping in gynecologic cancers

\begin{tabular}{|c|c|c|}
\hline & $\mathrm{NCCN}$ & ESGO \\
\hline $\begin{array}{l}\text { Endometrial } \\
\text { cancer }\end{array}$ & $\begin{array}{l}\text { - SLN mapping can be considered for apparently } \\
\text { uterine-confined disease when there is no metastasis } \\
\text { demonstrated by imaging studies or at exploration } \\
\text { - SLN mapping may also be used in high-risk histologies }\end{array}$ & $\begin{array}{l}\text { - SLN mapping is still experimental for apparently uterine-confined } \\
\text { disease and systematic lymphadenectomy is recommended }\end{array}$ \\
\hline Cervical cancer & $\begin{array}{l}\text { - SLN mapping can be considered in stage IA1 with } \\
\text { LVSI, IA2, IB1 and select IB2 cases } \\
\text { - SLN mapping can be used in tumors up to } 4 \mathrm{~cm} \text {, but } \\
\text { best detection rates are achieved in tumors }<2 \mathrm{~cm}\end{array}$ & $\begin{array}{l}\text { - SLN biopsy is an acceptable method of LN staging for stage IA1 } \\
\text { patients with LVSI and IA2 } \\
\text { - SLN biopsy in addition to BPLND is strongly recommended for } \\
\text { stage IB1-IIA1 patients. } \\
\text { - Intraoperative assessment of SLNs is also recommended for } \\
\text { stage IB1-IIA1 patients }\end{array}$ \\
\hline Vulvar cancer & $\begin{array}{l}\text { - SLN biopsy is an alternative standard of care approach } \\
\text { to inguinofemoral lymphadenectomy in select cases } \\
\text { - SLN biopsy can be used in patients with negative } \\
\text { clinical examination or imaging and unifocal tumors }<4 \\
\text { cm }\end{array}$ & $\begin{array}{l}\text { - (At least) unilateral SLN biopsy for tumors }>1 \mathrm{~cm} \text { from the } \\
\text { midline and bilateral SLN biopsy for tumors within } 1 \mathrm{~cm} \text { of the } \\
\text { midline should be performed for unifocal T1, }<4 \mathrm{~cm} \text { tumors in } \\
\text { case of no suspicious nodes preoperatively, and IFL should be } \\
\text { performed if SLN is not detected or positive }\end{array}$ \\
\hline Ovarian cancer & - Experimental & - Experimental \\
\hline
\end{tabular}




\section{Endometrial cancer}

SLN mapping is an alternative method for lymph node assessment in staging of apparently early-stage low-risk endometrial cancer patients when no metastasis is detected by imaging modalities or intraoperative exploration. Recent studies showed that SLN mapping can also be considered in high-risk histologies (type 2 endometrial cancer). To date, patients with macrometastasis and micrometastasis are treated in the same way, but adjuvant treatment of patients with ITCs is given according to primary tumor characteristics. Current data showed that removal of SLNs alone does not have a negative effect on oncological outcomes compared to complete lymphadenectomy, but more data are needed.

\section{Cervical cancer}

Both prospective and retrospective series have demonstrated that SLN mapping have high NPV in cervical cancer. However, SLN biopsy is completely reliable if bilateral SLNs are detected. SLN mapping may be used in tumors up to $4 \mathrm{~cm}$, but best detection rates are observed in tumors $<2 \mathrm{~cm}$.

\section{Vulvar cancer}

SLN biopsy is an option for inguinofemoral lymph node dissection in selected early-stage patients with unifocal tumors $<4 \mathrm{~cm}$ and in whom imaging and/or clinical examination reveals negative lymph nodes. Both prospective and retrospective series have demonstrated that SLN mapping has high NPV in vulvar cancer. For tumors $>4 \mathrm{~cm}$, SLN technique is both associated with reduced sensitivity and higher groin recurrences.

\section{Ovarian cancer}

SLN biopsy is an investigational approach in ovarian cancer. The best detection rates were observed with injections at the utero-ovarian and IP ligaments, or only at the IP ligament if hysterectomy had been performed before, just underneath the peritoneum. Pilot studies with limited number of patients showed moderate detection rate of SLNs in the pelvic region and high detection rate in the para-aortic region.

Peer-review: Externally peer-reviewed.

Conflict of Interest: No conflict of interest is declared by the authors.

Financial Disclosure: The authors declared that this study received no financial support.

\section{References}

1. National Comprehensive Cancer Network, NCCN clinical practice guidelines in oncology: uterine neoplasms, Version 1.2021. (Accessed on 7.11.2020) https://www.nccn.org/professionals/ physician_gls/pdf/uterine_blocks.pdf

2. National Comprehensive Cancer Network, NCCN clinical practice guidelines in oncology: cervical cancer, Version 1.2021. (Accessed on 7.11.2020) https://www.nccn.org/professionals/physician_gls/ pdf/cervical_blocks.pdf

3. National Comprehensive Cancer Network, NCCN clinical practice guidelines in oncology: vulvar cancer (squamous cell carcinoma), Version 2.2021. Accessed on 7.11.2020 https://www.nccn.org/ professionals/physician_gls/pdf/vulvar_blocks.pdf

4. Bodurtha Smith AJ, Fader AN, Tanner EJ. Sentinel lymph node assessment in endometrial cancer: a systematic review and metaanalysis. Am J Obstet Gynecol 2017; 216: 459-76.e10.

5. Rob L, Robova H, Halaska MJ, Hruda M, Skapa P. Current status of sentinel lymph node mapping in the management of cervical cancer. Expert Rev Anticancer Ther 2013; 13: 861-70.

6. Meads C, Sutton AJ, Rosenthal AN, Malysiak S, Kowalska M, Zapalska A, et al. Sentinel lymph node biopsy in vulval cancer: systematic review and meta-analysis. Br J Cancer 2014; 110: 2837 46.

7. Morice P, Leary A, Creutzberg C, Abu-Rustum N, Darai E. Endometrial cancer. Lancet 2016; 387: 1094-108.

8. Carlson JW, Kauderer J, Hutson A, Carter J, Armer J, Lockwood S, et al. GOG 244-The lymphedema and gynecologic cancer (LEG) study: Incidence and risk factors in newly diagnosed patients. Gynecol Oncol 2020; 156: 467-74.

9. Benedetti Panici P, Basile S, Maneschi F, Alberto Lissoni A, Signorelli M, Scambia G, et al. Systematic pelvic lymphadenectomy vs. no lymphadenectomy in early-stage endometrial carcinoma: randomized clinical trial. J Natl Cancer Inst 2008; 100: 1707-16.

10. ASTEC study group, Kitchener H, Swart AM, Qian Q, Amos C, Parmar MK. Efficacy of systematic pelvic lymphadenectomy in endometrial cancer (MRC ASTEC trial): a randomised study. Lancet 2009; 373: 125-36.

11. Mariani A, Webb MJ, Keeney GL, Haddock MG, Calori G, Podratz GC. Low-risk corpus cancer: is lymphadenectomy or radiotherapy necessary? Am J Obstet Gynecol 2000; 182: 1506-19.

12. Altin D, Taşkın S, Kahramanoglu I, Vatansever D, Tokgozoglu N, Karabük E, et al. Combination of sentinel lymph node mapping and uterine frozen section examination to reduce side-specific lymphadenectomy rate in endometrial cancer: a Turkish Gynecologic Oncology Group study (TRSGO-SLN-002). Int J Gynecol Cancer 2020; 30: 1005-11.

13. Vargas R, Rauh-Hain JA, Clemmer J, Clark RM, Goodman A, Growdon WB, et al. Tumor size, depth of invasion, and histologic grade as prognostic factors of lymph node involvement in endometrial cancer: a SEER analysis. Gynecol Oncol 2014; 133: 216-20.

14. Case AS, Rocconi RP, Straughn JM Jr, Conner M, Novak L, Wang W, et al. A prospective blinded evaluation of the accuracy of frozen section for the surgical management of endometrial cancer. Obstet Gynecol 2006; 108: 1375-9.

15. Gitas G, Proppe L, Alkatout I, Rody A, Kotanidis C, Tsolakidis D, et al. Accuracy of frozen section at early clinical stage of endometrioid endometrial cancer: a retrospective analysis in Germany. Arch Gynecol Obstet 2019; 300: 169-74.

16. Taşkın S, Şükür YE, Altın D, Ersöz CC, Turgay B, Kankaya D, et al. Laparoscopic near-infrared fluorescent imaging as an alternative option for sentinel lymph node mapping in endometrial cancer: A prospective study. Int J Surg 2017; 47: 13-7. 
17. Rossi EC, Kowalski LD, Scalici J, Cantrell L, Schuler K, Hanna RK, et al. A comparison of sentinel lymph node biopsy to lymphadenectomy for endometrial cancer staging (FIRES trial): a multicentre, prospective, cohort study. Lancet Oncol 2017; 18: 384-92.

18. Ballester M, Dubernard G, Lécuru F, Heitz D, Mathevet P, Marret $\mathrm{H}$, et al. Detection rate and diagnostic accuracy of sentinel-node biopsy in early stage endometrial cancer: a prospective multicentre study (SENTI-ENDO). Lancet Oncol 2011; 12: 469-76.

19. Taşkın S, Altin D, Vatansever D, Tokgozoglu N, Karabük E, Turan H, et al. Sentinel lymph node biopsy in early stage endometrial cancer: a Turkish gynecologic oncology group study (TRSGO-SLN-001). Int J Gynecol Cancer 2020; 30: 299-304.

20. Ducie JA, Eriksson AGZ, Ali N, McGree ME, Weaver AL, Bogani G, et al. Comparison of a sentinel lymph node mapping algorithm and comprehensive lymphadenectomy in the detection of stage IIIC endometrial carcinoma at higher risk for nodal disease. Gynecol Oncol 2017; 147: 541-8.

21. Zahl Eriksson AG, Ducie J, Ali N, McGree ME, Weaver AL, Bogani $\mathrm{G}$, et al. Comparison of a sentinel lymph node and a selective lymphadenectomy algorithm in patients with endometrioid endometrial carcinoma and limited myometrial invasion. Gynecol Oncol 2016; 140: 394-9.

22. Tanner EJ, Sinno AK, Stone RL, Levinson KL, Long KC, Fader AN. Factors associated with successful bilateral sentinel lymph node mapping in endometrial cancer. Gynecol Oncol 2015; 138: 542-7.

23. Martinelli F, Ditto A, Bogani G, Signorelli M, Chiappa V, Lorusso D, et al. Laparoscopic Sentinel Node Mapping in Endometrial Cancer After Hysteroscopic Injection of Indocyanine Green. J Minim Invasive Gynecol 2017; 24: 89-93.

24. Kim CH, Soslow RA, Park KJ, Barber EL, Khoury-Collado F, Barlin JN, et al. Pathologic ultrastaging improves micrometastasis detection in sentinel lymph nodes during endometrial cancer staging. Int $\mathrm{J}$ Gynecol Cancer 2013; 23: 964-70.

25. Fanfani F, Monterossi G, Di Meo ML, La Fera E, Dell'Orto F, Gioe A, et al. Standard ultra-staging compared to one-step nucleic acid amplification for the detection of sentinel lymph node metastasis in endometrial cancer patients: a retrospective cohort comparison. Int J Gynecol Cancer 2020; 30: 372-7.

26. Kost'un J, Pešta M, Sláma J, Slunéčko R, Vlasák P, Bouda J, et al. One-step nucleic acid amplification vs ultrastaging in the detection of sentinel lymph node metastasis in endometrial cancer patients. J Surg Oncol 2019; 119: 361-9.

27. Plante M, Stanleigh J, Renaud MC, Sebastianelli A, Grondin K, Gregoire J. Isolated tumor cells identified by sentinel lymph node mapping in endometrial cancer: Does adjuvant treatment matter? Gynecol Oncol 2017; 146: 240-6.

28. Bogani G, Casarin J, Maggiore ULR, Ditto A, Pinelli C, Dell'acqua A, et al. Survival outcomes in endometrial cancer patients having lymphadenectomy, sentinel node mapping followed by lymphadectomy and sentinel node mapping alone: Long-term results of a propensity-matched analysis. Gynecol Oncol 2020; 158: 77-83.

29. Multinu F, Ducie JA, Eriksson AGZ, Schlappe BA, Cliby WA, Glaser $\mathrm{GE}$, et al. Role of lymphadenectomy in endometrial cancer with nonbulky lymph node metastasis: Comparison of comprehensive surgical staging and sentinel lymph node algorithm. Gynecol Oncol 2019; 155: 177-85.

30. Buda A, Restaino S, Di Martino G, De Ponti E, Monterossi G, Dinoi G, et al. The impact of the type of nodal assessment on prognosis in patients with high-intermediate and high-risk ESMO/ESGO/ESTRO Group endometrial cancer. A multicenter Italian study. Eur J Surg Oncol 2018; 44: 1562-7.

31. Schiavone MB, Scelzo C, Straight C, Zhou Q, Alektiar KM, Makker $\mathrm{V}$, et al. Survival of Patients with Serous Uterine Carcinoma
Undergoing Sentinel Lymph Node Mapping. Ann Surg Oncol 2017; 24: 1965-71.

32. Selman TJ, Mann C, Zamora J, Appleyard TL, Khan K. Diagnostic accuracy of tests for lymph node status in primary cervical cancer: a systematic review and meta-analysis. CMAJ 2008; 178: 855-62.

33. Lécuru F, Mathevet P, Querleu D, Leblanc E, Morice P, Daraï E, et al. Bilateral negative sentinel nodes accurately predict absence of lymph node metastasis in early cervical cancer: results of the SENTICOL study. J Clin Oncol 2011; 29: 1686-91.

34. Altgassen C, Hertel H, Brandstadt A, Köhler C, Dürst M, Schneider A, et al. Multicenter validation study of the sentinel lymph node concept in cervical cancer: AGO study group. J Clin Oncol 2008; 26 : 2943-51.

35. Cibula D, Abu-Rustum NR, Dusek L, Slama J, Zikán M, Zaal A, et al. Bilateral ultrastaging of sentinel lymph node in cervical cancer: Lowering the false-negative rate and improving the detection of micrometastasis. Gynecol Oncol 2012; 127: 462-6.

36. Guani B, Balaya V, Magaud L, Lecuru F, Mathevet P. The clinical impact of low-volume lymph nodal metastasis in early-stage cervical cancer: the Senticol 1 and Senticol 2 Trials. Cancers 2020; 12: 1061.

37. Woelber L, Eulenburg C, Choschzick M, Kruell A, Petersen C, Gieseking $\mathrm{F}$, et al. Prognostic role of lymph node metastases in vulvar cancer and implications for adjuvant treatment. Int J Gynecol Cancer 2012; 22: 503-8.

38. Grimm D, Prieske K, Mathey S, Kuerti S, Burandt E, Schmalfeldt B, et al. Superficially invasive stage IA vulvar squamous cell carcionomatherapy and prognosis. Int J Gynecol Cancer 2019; 29: 466-73.

39. Homesley HD, Bundy BN, Sedlis A, Yordan E, Berek JS, Jahshan A, et al. Prognostic factors for groin metastasis in squamous cell carcinoma of the vulva (a Gynecologic Oncology Group study). Gynecol Oncol 1993; 49: 279-83.

40. Brincat MR, Muscat Baron Y. Sentinel lymph node biopsy in the management of vulvar carcinoma: an evidence-based insight. Int J Gynecol Cancer 2017; 27: 1769-73.

41. Covens A, Vella ET, Kennedy EB, Reade CJ, Jimenez W, Le T. Sentinel lymph node biopsy in vulvar cancer: Systematic review, meta-analysis and guideline recommendations. Gynecol Oncol 2015; 137(2): 351-61.

42. Levenback CF, Ali S, Coleman RL, Gold MA, Fowler JM, Judson PL, et al. Lymphatic mapping and sentinel lymph node biopsy in women with squamous cell carcinoma of the vulva: a gynecologic oncology group study. J Clin Oncol 2012; 30: 3786-91.

43. Fotopoulou C, Ind T, Baldwin P, Crawford R, Devaja O, Dobbs S, et al. Sentinel lymph node consensus document of the British Gynaecological Cancer Society for endometrial, vulvar, and cervical cancers. Int J Gynecol Cancer 2019; 29: 1348-50.

44. Van der Zee AG, Oonk MH, De Hullu JA, Ansink AC, Vergote I, Verheijen RH, et al. Sentinel node dissection is safe in the treatment of early-stage vulvar cancer. J Clin Oncol 2008; 26: 884-9.

45. Te Grootenhuis NC, van der Zee AG, van Doorn HC, van der Velden J, Vergote I, Zanagnolo V, et al. Sentinel nodes in vulvar cancer: Long-term follow-up of the GROningen INternational Study on Sentinel nodes in Vulvar cancer (GROINSS-V) I. Gynecol Oncol 2016; 140: 8-14.

46. Oonk MHM, Slomovitz B, Baldwin P, van Doorn H, van der Velden $\mathrm{J}$, de Hullu $\mathrm{J}$, et al. Radiotherapy instead of inguinofemoral lymphadenectomy in vulvar cancer patients with a metastatic sentinel lymph node: results of GROINSS-V II. Int J Gynecol Cancer 2019; 29(Suppl4): A13.

47. Morice P, Joulie F, Camatte S, Atallah D, Rouzier R, Pautier P, et al. Lymph node involvement in epithelial ovarian cancer: analysis of 276 pelvic and paraaortic lymphadenectomies and surgical implications. J Am Coll Surg 2003; 197: 198-205. 
48. Cass I, Li AJ, Runowicz CD, Fields AL, Goldberg GL, Leuchter RS et al. Pattern of lymph node metastases in clinically unilateral stage I invasive epithelial ovarian carcinomas. Gynecol Oncol 2001; 80: $56-61$.

49. Timmers PJ, Zwinderman K, Coens C, Vergote I, Trimbos JB. Lymph node sampling and taking of blind biopsies are important elements of the surgical staging of early ovarian cancer. Int J Gynecol Cancer 2010; 20: 1142-7.

50. Dell'Orto F, Laven P, Delle Marchette M, Lambrechts S, Kruitwagen R, Buda A. Feasibility of sentinel lymph node mapping of the ovary: a systematic review. Int J Gynecol Cancer 2019; 29: 1209-15.
51. Lago V, Bello P, Montero B, Matute L, Padilla-Iserte P, Lopez S, et al. Sentinel lymph node technique in early-stage ovarian cancer (SENTOV): a phase II clinical trial. Int J Gynecol Cancer 2020; 30: $1390-6$.

52. Uccella S, Nero C, Vizza E, Vargiu V, Corrado G, Bizzarri N, et al. Sentinel node biopsy in early-stage ovarian cancer: preliminary results of a prospective multicentre study (SELLY). Am J Obstet Gynecol 2019; 221: 324.e10. 